Meta

Journal des tradlucteurs

Translators' Journal

\title{
Réflexions sur l'interprétation
}

\section{Paul V. Hendrickx}

Volume 14, numéro 2, juin 1969

URI : https://id.erudit.org/iderudit/003044ar

DOI : https://doi.org/10.7202/003044ar

Aller au sommaire du numéro

Éditeur(s)

Les Presses de l'Université de Montréal

ISSN

0026-0452 (imprimé)

1492-1421 (numérique)

Découvrir la revue

Citer cet article

Hendrickx, P. V. (1969). Réflexions sur l'interprétation. Meta, 14(2), 83-88.

https://doi.org/10.7202/003044ar d'utilisation que vous pouvez consulter en ligne.

https://apropos.erudit.org/fr/usagers/politique-dutilisation/ 


\section{réflexions sur l'interprétation}

I. Il faut regretter le manque de compréhension de la part du public et de certains employeurs en présence des problèmes et des conditions de travail de l'interprète ${ }^{1}$. Lors de la «British Week » à Bruxelles en septembre dernier, des organisateurs de conférences s'étonnaient du «manque de compétence» de certains interprètes professionnels. Les performances des interprètes en simultanée, lors d'un débat sur les communications interspatiales par exemple, n'étaient guère brillantes. À l'examen cependant, il s'avérait que les organisateurs ne s'étaient même pas donné la peine de renseigner à l'avance les interprètes sur la nature précise du débat et qu'on exigeait d'eux des prestations de plusieurs heures d'affilée, sans relais.

Le public semble supposer qu'un interprète, par le fait même qu'il est professionnel, est d'office versé dans toutes les disciplines imaginables, et qu'il est capable d'un effort de concentration constant et illimité. En outre, le public semble ignorer totalement qu'il ne suffit pas de connaitre des langues pour être traducteur et qu'un bon traducteur n'est pas nécessairement un bon interprète. Il est urgent donc que le public soit mieux informé.

Ma seconde considération se rapporte aux méthodes d'enseignement de la traduction simultanée.

L'année passée, lors de l'assemblée générale de l'A.I.T., M. W. Keiser a insisté à juste titre sur la nécessité pour l'interprète diplômé d'être immédiatement utilisable. Par ailleurs, Jean Herbert a écrit que le niveau de l'interprète diplômé doit être tel que le lendemain de l'examen, le professeur-interprète soit prêt à envoyer l'impétrant à sa place en salle de conférences. Or, nous savons tous que la méthodologie de la traduction n'en est qu'à ses débuts, et que les méthodes d'enseignement sont souvent élaborées empiriquement, sans parler des cas où une méthode appropriée fait entièrement défaut.

1. Cf. Thérèse Nilski, "Translators and Interpreters - Siblings or a Breed Apart ", META, XII, 2 (juin 1967). 
On peut, bien sûr, estimer que le don d'interprétation est inné et ne s'acquiert pas. Toutefois, la création de tant d'écoles d'interprétariat semble démontrer que tel n'est pas l'avis de tous et que la formation «sur le tas » s'est avérée insuffisante. Par conséquent, il est urgent de mettre tout en œuvre pour que tous les aspects de la formation d'interprètes soient étudiés systématiquement, afin de trouver une didactique scientifiquement justifiée.

Cela nécessite la collaboration de tous: des gouvernements et des écoles, des institutions internationales et des entreprises privées, des interprètes chevronnés et des pédagogues. En effet, pour former de bons interprètes, il ne suffit pas qu'un gouvernement ou un mécène quelconque accorde des subsides, qu'une école décrète des conditions d'admission, qu'un comité définisse les modalités de constitution d'un jury. La question n'est pas de savoir qui, des enseignants ou des interprètes, est le mieux qualifié pour occuper une chaire dans une école d'interprétariat. La question est de trouver les bonnes méthodes d'enseignement et de formation, et de désigner ensuite les personnes capables d'appliquer ces méthodes avec succès.

C'est alors, et alors seulement, que nous aurons la certitude que nos étudiants seront «immédiatement utilisables» et que nous pourrons les envoyer en toute tranquillité à notre place à la salle de conférences.

II. C'est sans doute dans le domaine de l'enseignement de la traduction simultanée que l'absence d'une didactique scientifiquement établie se fait sentir avec le plus d'acuité. Rares sont les ouvrages où ce sujet est quelque peu approfondi et les vues des enseignants et des interprètes professionnels sont en ce domaine extrêmement divergentes.

Deux thèses sont cependant acceptées par la plupart des intéressés: 1) l'interprète ne peut pas tout savoir, mais doit être capable de tout trouver; 2) dans les cours de traduction simultanée, l'activité principale consiste à enseigner les techniques de la traduction simultanée et non pas les langues, ni la traduction proprement dite.

De la première thèse, il résulte qu'il faut apprendre aux futurs interprètes à trouver et à assimiler très rapidement des quantités de termes assez considérables. Il ne s'agit pas de faire ingurgiter à l'étudiant des listes de mots, mais de mettre au point une méthode d'acquisition rapide de connaissances terminologiques.

D'autre part, si l'on accepte la seconde proposition, il faut bien admettre que les méthodes employées actuellement dans la plupart des écoles n'insistent pas assez sur la technique pure de la traduction simultanée. Généralement, la leçon comporte grosso modo deux parties. La première - parfois appelée séminaire permet aux étudiants de se familiariser avec le sujet et le vocabulaire. À cet effet, on procède par paragraphes à des traductions provisoires, on limite le débit de la lecture, on observe une pause après chaque phrase, on discute au passage les traductions proposées, ou bien encore, ainsi que nous le voyons dans l'excellent livre de M. Henri Van Hoof ${ }^{2}$, les textes à traduire sont travaillés par phrases courtcs ne comportant qu'une seule idée. Souvent même, les textes sont traduits en consécutive.

2. Henri Van Hoof, Théorie et pratique de l'interprétation, Munich, Max Hueber, 1963. 
La seconde partie de la leçon - parfois appelée laboratoire - consiste à faire traduire le même texte, ou des textes très analogues, en simultanée. Elle est suivie ou accompagnée de commentaires critiques du professeur.

Cette façon de procéder contribue sans aucun doute grandement à la formation du traducteur, mais dans la pratique, l'exercice se limite en fin de compte trop souvent à une répétition d'une traduction plus ou moins apprise d'avance. De toute façon, la leçon comporte trop d'éléments étrangers à l'apprentissage de la technique de la traduction simultanée proprement dite. Sans doute apprend-on aux étudiants à réagir très vite, et à mémoriser rapidement, mais les principes de la simultanée ne sont mis en pratique que subséquemment. Ce qui devrait être la part du lion se réduit à fort peu de chose. Il en résulte, à la fin de la période de formation, une connaissance insuffisante de la technique de la simultanée proprement dite, ce qui favorise la tendance naturelle à se lancer dans le «mot à mot irréfléchi, qui est considéré unanimement comme néfaste, sauf dans des cas exceptionnels (par exemple une succession de chiffres, où il n'y a rien à « comprendre »).

On peut estimer qu'en exerçant sa profession, l'interprète se rendra bien vite compte qu'il faut comprendre le sens et saisir la portée des mots avant de les traduire - même en simultanée - et que, par conséquent, la nécessité d'un recul relativement important se fera sentir de toute façon dès que le sujet du discours est dialectique, abstrait ou affectif. Mais c'est cela précisément qu'il convient de faire comprendre et de faire acquérir le plus tôt possible. Il apparaît avec la plus grande évidence qu'il faut veiller dès le début de la formation à ce que les étudiants appliquent les techniques correctes qui permettent aux moins doués d'obtenir des résultats satisfaisants et aux très doués de réussir des performances qu'ils n'auraient jamais pu réaliser sans cette formation de base.

III. Dès lors, le problème fondamental est de savoir quelle est la technique la plus efficace et par quels moyens nous pouvons la faire appliquer. Pour résoudre ce problème, il faut en premier lieu déterminer avec précision en quoi la traduction simultanée se différencie des autres formes de traduction qu'on enseigne dans d'autres cours.

Les résultats des recherches sur le mécanisme cérébral de la traduction ne sont guère concluants et les avis ne concordent pas toujours, mais il est permis de penser que la traduction orale comporte en gros quatre phases: 1) l'audition: écouter, capter des phonèmes; 2) l'idéation: entendre, transformer ces phonèmes en concept; 3) la traduction: transformer le concept en phonèmes correspondants d'une autre langue; 4) la phonation: articuler, émettre ces nouveaux phonèmes.

Il n'est pas établi à quel stade de la simultanée se fait dans le cerveau la rétention et l'association des renseignements reçus; il est même probable que dans certains cas la deuxième et la troisième phases se confondent (automatisme verbal). Par ailleurs, il est possible que le processus d'association dans la conception et dans l'expression ne soit pas identique pour toutes les formes de la traduction. Mais il est certain que, contrairement à ce qui se passe en consécutive, la phase 1 - et peut-être les phases 2 et 3 également - reprend en simultanée avant que la phase 4 ne soit terminée. 
Voilà ce qui constitue l'élément spécifique de la traduction simultanée.

Il en résulte qu'aux cours de simultanée, l'activité principale doit consister à apprendre ou à développer la dissociation de l'attention qui rend le décalage possible, qui permet le recul nécessaire à une traduction réfléchie. C'est ce que Danica Seleskovitch ${ }^{3}$ appelle la concentration sur la double activité, entendre et parler; ou encore: audition et rétention d'une part, traduction et phonation d'autre part .

Plusieurs moyens ont déjà été suggérés pour atteindre cet objectif principal, mais aucune méthode ne semble avoir été soumise à un examen scientifique. Danica Seleskovitch propose deux sortes d'exercices préalables à la reconstitution complète du sens en simultanée: 1) faire compter à rebours, dans la langue maternelle, tout en écoutant et en suivant le sens d'un texte simple présenté dans une autre langue; 2) le reportage: l'étudiant en cabine parle en même temps que l'orateur, mais ne traduit pas encore; il explique dans ses propres termes ce dont il s'agit, comme un reporter de radio racontant un match de football.

Ces exercices pourraient être variés à l'infini, par exemple en remplaçant le compte à rebours par l'énumération des lettres de l'alphabet, ou la récitation des tables de multiplication ou d'un texte appris par cœur. On pourrait demander à l'étudiant d'écrire une lettre, de répondre à des questions écrites, de converser, tout en écoutant un discours dont il devra après faire une consécutive résumée.

Contrairement à ce qu'on pourrait croire, ces exercices ne sont pas une perte de temps. Non seulement ils développent la capacité de dissociation de l'attention, mais ils donnent aussi plus d'assurance à l'étudiant puisqu'ils lui permettent de vaincre en partie la principale difficulté technique avant qu'il ne se lance dans la traduction simultanée complète. N'est-ce pas une des règles fondamentales de la didactique qu'il ne faut jamais enseigner deux choses en même temps?

IV. Cependant, ce genre d'exercices ne permet pas de mesurer avec précision les résultats partiels obtenus. De ce fait, le dosage et la gradation des difficultés, éléments tellement souhaitables dans l'enseignement, sont assez difficiles. C'est pourquoi on pourrait envisager l'emploi du système suivant.

Le professeur dit à haute voix et à un rythme à peu près égal à la vitesse normale de la parole, des séries de chiffres. Il invite les élèves à les répéter en augmentant progressivement le recul. $\mathrm{Si}$, par exemple, la série commence par $7,4,8,6 \ldots$, il demandera de commencer la répétition à la lecture du chiffre 4 , puis à 8 , puis à 6 . Il convient de veiller à ce que la répétition se fasse au même rythme que la lecture, de façon à conserver le même décalage jusqu'à la fin de la série. En outre, il ne faut pas exiger un recul plus grand avant que les élèves ne soient capables de supporter, jusqu'au bout et sans se tromper, un recul moins grand.

Lorsque la classe est capable de répéter, puis de traduire sans erreurs 5 ou 6 séries de 20 chiffres avec un recul de 3 ou 4 unités par exemple, on procède de même avec des séries de nombres allant progressivement de 2 à 6 chiffres. On aura soin de ne pas exiger l'impossible, mais d'autre part on

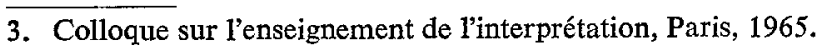


s'assurera d'avoir atteint le maximum de recul avant de passer au groupe suivant. Aussi faut-il présenter constamment des chiffres différents afin d'éviter la mémorisation qui fausserait les résultats. Sans doute pourrait-on procéder de même avec de petites opérations d'arithmétique, etc.

L'avantage incontestable des chiffres réside dans le fait qu'ils constituent des unités de longueur identique, qui présentent toutes à peu près les mêmes difficultés de rétention et de traduction, ce qui permet un dosage rigoureux et une progression systématique.

Ensuite, on pourrait substituer aux chiffres des séries de mots en langue étrangère dont la traduction présente progressivement des difficultés accrues, puis des groupes de mots, puis des phrases entières et enfin, de textes continus.

Dans tous les cas, il convient de veiller à ce que le plus grand décalage possible soit conservé jusqu'au bout. D'autre part, il est de la plus grande importance que la tâche proposée aux élèves ne présente en aucun moment des difficultés insurmontables, ni de traduction, ni de rétention. La méthode qui consiste à faire "patauger » les étudiants, sous prétexte que les meilleurs se débrouillent toujours, va à l'encontre des principes didactiques élémentaires.

Toutefois, il n'est pas recommandable de réduire la difficulté en « préparant » les exercices. C'est une erreur assez courante que de «travailler» le même texte (ou des textes très semblables) à plusieurs reprises et sous des angles différents, puis de se leurrer de l'illusion qu'on fait des progrès en simultanée, alors qu'on a fait des progrès uniquement en rapidité de traduction. Il faut toujours travailler avec de la matière nouvelle, mais choisie de telle façon que les difficultés ne s'accumulent pas.

Il va sans dire que cette méthode n'exclut pas d'autres exercices, tels que la traduction à vue, qui est un excellent moyen d'augmenter la rapidité de réaction, mais qui n'est cependant pas idéale, car le futur interprète en simultanée doit apprendre le plus tôt possible à se passer de la présentation visuelle des éléments à traduire. En effet, il est établi que les conditions de la perception, de la transmission et de l'association des mots entendus ne sont pas les mêmes que celles de la perception, de la transmission et de l'association des mots lus. Comme ces activités se situent dans des régions ou des centres différents du cerveau, un exercice qui convient au développement de l'une de ces facultés ne favorise que peu ou pas le développement de l'autre.

D'autre part, la traduction à vue est une pratique qu'on retrouve assez souvent en conférence!

V. Il convient enfin de parler du choix des sujets à traiter, car il n'est pas toujours facile de juger du degré de difficulté d'une matière. On a trop souvent tendance à juger un texte uniquement en fonction des difficultés lexicologiques, alors que tant d'autres facteurs devraient être examinés. Pour Danica Seleskovitch le problème se présente de la façon suivante:

Il me semble que le contenu des exposés doit être le domaine réservé à l'inspiration, au talent et à l'intérêt de chaque enseignant, mais il serait intéressant de discuter de la progression des difficultés en interprétation, qui me semble être la suivante: 
LANGUES: forme descriptive, forme argumentative, forme rhétorique.

SUJET: concret, abstrait, affectif.

TECHNIQUES D'INTERPRÉTATION: consécutive en langue A, simultanée en A, consécutive en $\mathrm{B}$.

Théoriquement, sur le plan de l'enseignement de la technique de l'interprétation, les sujets descriptifs techniques sont plus faciles à transposer que les autres, à condition bien entendu de connaître ou d'avoir préparé la terminologie.

Le choix des sujets ou des textes doit se faire en tenant compte de toutes ces considérations, mais ici encore, il s'agit de dégager la difficulté d'un discours au point de vue spécifique de la traduction simultanée.

Sur ce plan, le critère dominant semble être la compatibilité des langues et des styles. En effet, les difficultés provenant de la spécialisation du vocabulaire ou de la complexité du fond se manifestent tout autant en consécutive qu'en simultanée. En simultanée cependant, un discours est d'autant plus facile à traduire que le langage employé peut être calqué dans la langue d'arrivée.

C'est pourquoi on pourrait envisager au début de faire usage de bonnes traductions, c'est-à-dire de faire traduire en français, par exemple la version anglaise d'un discours qui a été prononcé à l'origine en français. Dans ce cas les structures seront malgré tout assez semblables et cela constitue un moyen admissible pour limiter les difficultés.

Est-il besoin d'ajouter encore qu'il est recommandable de s'efforcer de présenter l'objet à interpréter sous une forme aussi orale que possible? Malgré les inconvénients de la traduction à partir d'enregistrements sur bandes, il serait utile de constituer dans les écoles des «bibliothèques orales 》 ou "sonothèques", composées d'enregistrements pris sur le vif. Encore faut-il veiller à enregistrer la parole libre et non pas la lecture d'un texte écrit d'avance. Même les conférences présentées à la radio ne sont pas toujours recommandables, car le style en est souvent trop artificiel et le contenu trop concentré.

En conclusion, il apparaît avec évidence qu'il faut s'efforcer à tous les stades des cours, généralement trop peu nombreux, consacrés à l'interprétation simultanée, à se concentrer sur l'apprentissage des techniques pures de la traduction simultanée. Les résultats ne seront peut-être pas aussi spectaculaires au début, mais ainsi il ne sera plus nécessaire, par après, de recourir à des artifices pour rattraper l'orateur, et la qualité de l'interprétation s'en trouvera sensiblement améliorée.

Paul V. Hendrickx 